\title{
Vigilancia epidemiológica de la infección del sitio quirúrgico en ortopedia
}

\author{
Epidemiological surveillance of surgical site infection in orthopedics \\ Rafael Franco-Cendejas*
}

*Jefe de División de Infectología. Instituto Nacional de Rehabilitación «Luis Guillermo Ibarra Ibarra».

Correspondencia: Dr. M. en C. Infectólogo Rafael Franco-Cendejas. Av. México-Xochimilco No. 289, Col. Arenal de Guadalupe, 14389, Alcaldía Tlalpan, Ciudad de México, México. Correo electrónico: raffcend@yahoo.com

\section{RESUMEN}

La infección del sitio quirúrgico representa un problema importante para los sistemas de salud de cualquier país. Es la principal complicación a nivel general de los pacientes sometidos a un procedimiento quirúrgico. La vigilancia de la presentación de dichos problemas es de suma importancia, esto con la finalidad de identificar y prevenir su presentación. Hay varios factores que se deben tomar en cuenta, uno de los principales es identificar correctamente dichas complicaciones mediante una definición estandarizada, con el objetivo de identificarlas y clasificarlas. Se deben tener sistemas de vigilancia adecuadamente estructurados para detectar correctamente el número y el tipo de infecciones. Asimismo, se debe mantener informado al personal de asistencia, directivo y usuario de los resultados de dicha vigilancia.

Palabras clave: Infección sitio quirúrgico, infección superficial, infección profunda, infección de órganos/espacios, vigilancia postquirúrgica.

\section{ABSTRACT}

Surgical site infection represents a major problem for the health systems of any country. They are the main complication at the general level of patients undergoing a surgical procedure. SSI Surveillance of such problems is of utmost importance in order to identify and prevent their presentation. There are several factors that must be taken into account, one of the main ones is to correctly identify these complications using a standardized definition, in order to identify and classify them. There must be properly structured surveillance systems to correctly detect the number and type of infections. Assistance personnel, managers and users must be kept informed of the results of said surveillance.

Keywords: Surgical site infection, superficial infection, deep infection, organ/space infection, surgical surveillance. 


\section{INTRODUCCIÓN}

La morbilidad, la mortalidad y el costo para los servicios de salud de las infecciones del sitio quirúrgico son enormes. Sólo en los Estados Unidos se estima que se realizan 50 millones de procedimientos quirúrgicos cada año con casi $40 \%$ de los pacientes mayores de 65 años. ${ }^{1}$ Las infecciones del sitio quirúrgico son la tercera infección nosocomial notificada con mayor frecuencia y representan de 14 a $16 \%$ de todas las infecciones nosocomiales entre sujetos hospitalizados. ${ }^{1}$ Entre los pacientes quirúrgicos, las infecciones del sitio quirúrgico (ISQ) representaron la tercera parte de las infecciones nosocomiales y éstas fueron las más comúnmente encontradas, ${ }^{2}$ lo cual puede variar de acuerdo a las características de cada hospital. Dos tercios de las ISQ se limitaron a la incisión quirúrgica y un tercio involucró órganos o espacios a los que se accedió durante el procedimiento quirúrgico. Asimismo, se ha relacionado con mortalidad hasta en $1.3 \%$ de los casos. ${ }^{3}$

Estos datos resaltan el enorme problema que enfrentan los servicios de salud en la actualidad e indican la importancia de intentar disminuir la incidencia de la ISQ.

Freeman, ${ }^{4}$ en una revisión de métodos de vigilancia de la ISQ comenta que, para que dichos métodos sean significativos, los sistemas de vigilancia deben ser más sólidos en su recopilación de datos y más sensibles en su identificación de tendencias y brotes, incluso deben contemplar parámetros como la resistencia bacteriana. El cómo se realiza la recopilación de la información y el momento en que se lleva acabo el registro de los datos también tiene un impacto significativo en las cifras; por ejemplo, un estudio que comparó las tasas de ISQ en cirugía cardiotorácica mostró discrepancias significativas en las tasas registradas de diferentes métodos utilizados; además, se observó que sólo $21 \%$ de ISQ se había registrado, inclusive al evaluar varias fuentes para recopilar la información. ${ }^{5}$ Por ello, es de suma importancia obtener datos que tengan utilidad y validez, ya que los datos de vigilancia deben ser capaces de identificar grupos, rastrear tendencias, evaluar la efectividad de las intervenciones y proporcionar resultados oportunos. ${ }^{6}$

Los elementos básicos para que un programa de prevención de ISQ sea exitoso incluyen actividades intensivas de vigilancia y una retroalimentación periódica de las tasas de ISQ. ${ }^{7}$ Además, hasta el momento no hay evidencia que genere un cambio en el informe mensual de las tasas comparado con los trimestrales o semestrales. ${ }^{8}$

Al dividir un programa de vigilancia de ISQ es necesario tener en cuenta las definiciones de infección, recopilación de datos, manejo y análisis, presentación de resultados y qué pacientes deben incluirse. ${ }^{9}$

El control de infecciones es un trabajo en equipo; sin embargo, hay involucrados que sin su participación podría no funcionar o cambiar las cosas, tal es el caso de los integrantes de la Unidad de Vigilancia Epidemiológica, los infectólogos, el laboratorio de microbiología, los ortopedistas y los directivos del 
hospital. De acuerdo con el número de personal de control de infecciones que se encuentre asignado, de la disposición del personal clínico que desee involucrarse, la vigilancia tendrá que adaptarse en lo posible cumpliendo metas fijas y pequeñas en lugar de arriesgar la integridad de la estrategia de vigilancia total al ser demasiado ambicioso.

\section{DEFINICIONES}

Uno de los puntos más importantes para la vigilancia de las ISQ es que se utilicen definiciones estandarizadas; de otra forma, se pueden obtener e informar resultados inexactos y sesgados. Información relevante que ayuda a comparar los datos y a evaluar el comportamiento a lo largo del tiempo son los canales endémicos (por lo cual es crucial que las definiciones se mantengan sin cambios para que se puedan establecer las tasas de referencia de las ISQ), el riesgo de los pacientes de desarrollarla y si hemos hecho alguna intervención, todo ello con la finalidad de evaluar los resultados de las mismas e importantemente las comparaciones interhospitalarias.

La definición más utilizada de ISQ es la empleada por el sistema National Nosocomial Infections Surveillance (NNIS) de los Centers for Disease Control (CDC).$^{10}$ Su estandarización es cada vez más importante a medida que las tasas de ISQ se interpreten como una medida de la calidad de la atención al paciente.

La definición actual ahora clasifica las ISQ en incisional superficial o profundas involucrando órganos o espacio. ${ }^{11}$ Las ISQ incisionales superficiales a su vez se subdividen en ISQ incisionales superficiales (que involucran sólo piel y tejido subcutáneo) e ISQ incisionales profundas (que involucran tejidos blandos más profundos). Las ISQ profundas involucran cualquier parte de la anatomía que no sean las capas incisas de la pared corporal, cuando se abrió o manipuló durante un procedimiento quirúrgico y además pueden incluir la introducción de algún dispositivo.

\section{DEFINICIÓN DE INFECCIÓN DEL SITIO QUIRÚRGICO}

Toda ISQ se define como aquélla relacionada con el procedimiento quirúrgico que se produce en la incisión quirúrgica o en su contorno, durante los primeros 30 o 90 días del postoperatorio dependiendo del procedimiento quirúrgico realizado.

Es de suma importancia conocer las siguientes definiciones, ya que muchas veces ocurren sesgos en la interpretación clínica de valoración de los pacientes al pensar que la presencia de eritema o la salida de exudado pueden ser considerados como criterios únicos en la presencia de un foco infeccioso. ${ }^{11}$

\section{INFECCIÓN SUPERFICIAL}

La infección ocurre en el lugar de la incisión que afecta sólo al tejido cutáneo y subcutáneo, se produce en los 30 días posteriores a la intervención (siendo el 
día uno el día de la intervención quirúrgica) y al menos debe cumplir uno de los siguientes criterios:

a. Drenaje purulento procedente de la incisión superficial.

b. Microorganismo aislado de un cultivo (que sea de una muestra clínica para fines diagnósticos o terapéuticos, y no el resultado de una búsqueda activa de casos) obtenido asépticamente de líquido o tejido procedente de la incisión superficial.

c. Incisión superficial que es abierta deliberadamente por el cirujano o médico responsable ante la sospecha de infección y no realización de cultivo u otra prueba microbiológica. Y al menos uno de los siguientes signos o síntomas de infección: dolor, hipersensibilidad al tacto o a la presión, inflamación localizada (calor, tumefacción, eritema). El hallazgo de un cultivo u otra prueba microbiológica negativo no hace válido este criterio.

d. Diagnóstico de ISQ incisional superficial por el médico responsable.

\section{Existen dos tipos de infección superficial}

Incisional superficial primaria: infección superficial localizada en la incisión principal en un paciente sometido a una cirugía con una o más incisiones (como la incisión en tórax realizada durante un bypass coronario que utiliza un injerto procedente de otra parte del cuerpo).

Incisional superficial secundaria: infección superficial localizada en la incisión no principal en un paciente sometido a una cirugía en la que se ha practicado más de una incisión (como la incisión en la pierna, realizada para tomar un injerto, necesario en un bypass coronario.

\section{INFECCIÓN PROFUNDA}

La infección se produce en el lugar de la intervención que afecta a tejidos blandos profundos de la incisión (fascia y paredes musculares), se produce en los 30 días posteriores a la intervención o en los 90 días posteriores (siendo el día uno el día de la intervención quirúrgica) y, al menos, debe cumplir uno de los siguientes criterios:

a. Drenaje purulento de la zona profunda de la incisión.

b. Dehiscencia espontánea de la herida o apertura deliberada por el cirujano o médico responsable ante la sospecha de infección, microorganismo aislado de un cultivo o de un examen microbiológico diferente al cultivo (que sea de una muestra clínica para fines diagnósticos o terapéuticos, y no el resultado de una búsqueda activa de casos), o no se haya realizado cultivo ni otra prueba microbiológica. Asimismo, el paciente debe presentar al menos uno de los siguientes síntomas: fiebre $>38^{\circ} \mathrm{C}$, dolor localizado o hipersensibilidad al tacto o a la presión. El hallazgo de un cultivo negativo u otra prueba microbiológica negativa no hace válido este criterio. 
c. Absceso u otro signo de infección en la incisión profunda detectado por examen macroscópico anatómico, histopatológico o estudio de imagen.

\section{INFECCIÓN DE ÓRGANOS Y ESPACIOS}

La ISQ de órganos y espacio involucra cavidades manipuladas durante la cirugía y ocurre dentro de los 30 días posteriores a la operación o 90 días después si se deja un implante en su lugar. La infección parece estar relacionada con la cirugía, pero no involucra la parte superficial. Asimismo, debe estar presente uno de los siguientes criterios:

a. Diagnóstico por parte de un médico o cirujano.

b. Evidencia de infección (por ejemplo, absceso) que se identifique durante la evaluación, el manejo o el examen histopatológico o radiológico.

c. Cultivo obtenido asépticamente (tejido o fluido) que demuestra microorganismos causantes.

d. Drenaje purulento de la cavidad o de un dispositivo colocado.

\section{MÉTODOS}

\section{Obtención de los datos}

Es muy importante que se obtenga la información tanto pasivamente (esperando que nos avisen o llegue la información) como activamente (realizando búsqueda personalizada en el laboratorio, consulta, hospitalización, expedientes, etcétera) acerca de los potenciales casos. Se deben tener tanto variables sociodemográficas como los potenciales factores de riesgo, variables relacionadas con la hospitalización, el evento quirúrgico, el proceso infeccioso y lo microbiológico.

Tipo de vigilancia y seguimiento

Se han descrito varios tipos de métodos de vigilancia de infecciones nosocomiales con diferente sensibilidad y especificidad (incluyendo revisión de Kardex de enfermería, reportes microbiológicos, uso de antimicrobianos, valoración de temperaturas, vigilancia de factores de riesgo, llamadas telefónicas al laboratorio y vigilancia de indicaciones o medidas mixtas). Incluso se han comparado estudios en los cuales el personal específico de epidemiología es mucho más sensible en la identificación de la ISQ que el personal de asistencial habitual, ${ }^{12}$ por lo que se debe promover y apoyar a los trabajadores indicados para realizar esta actividad.

Se realizará una vigilancia activa, siendo el personal de la Unidad de Vigilancia Epidemiológica Hospitalaria, designado en cada hospital, los responsables de identificar diariamente a los pacientes sometidos al procedimiento quirúrgico a vigilar y de realizar un seguimiento periódico (cada 24 o 48 horas) de estos sujetos con el fin de detectar la aparición de ISQ. Cada hospital adaptará la 
vigilancia a sus características específicas. También se recomienda combinar con vigilancia pasiva para la evaluación de las infecciones, la cual está dada por los reportes/avisos que hacen a diario las enfermeras o médicos identificando los casos, o bien recibiendo los resultados de cultivos de los laboratorios de microbiología.

Se sugiere realizar una vigilancia de los eventos ortopédicos por paciente, independientemente del tipo de cirugía que se realice, y aquellas cirugías que incluyan colocación de algún dispositivo ortopédico se deban vigilar igual que aquéllas con colocación de una prótesis.

\section{Periodo de vigilancia}

En el caso de los padecimientos ortopédicos, se vigilarán durante 30 días aquéllos en los que no se introdujo material y en los que sí hubo colocación de algún dispositivo ortopédico por al menos 90 días. Si no hay detección de casos en los 90 días se sugiere continuar la evaluación de los procedimientos en aquellos casos en los que hubo colocación de algún dispositivo ortopédico; esta valoración se podrá seguir hasta por un año, ya que se ha observado que puede haber falta en la detección de algunas de estas infecciones, tal como lo demuestra un estudio en el que se observó que por la reducción en el tiempo de seguimiento se pueden perder de 8.8 a $25.1 \%$ de los diagnósticos en las infecciones de prótesis de cadera y rodilla, respectivamente..$^{13}$

\section{Variables de estudio}

Se recogerán variables relativas al hospital y a la unidad, al paciente y a la hospitalización, a la intervención quirúrgica y a la infección y las relativas a los microorganismos aislados y a las resistencias.

\section{Reporte de la información}

Se debe realizar un reporte mensual para el comité de infecciones intrahospitalarias, en el cual se discutirán las tasas de cada servicio y de cada procedimiento. Se sugiere que se haga de manera mensual, con la finalidad de identificar tempranamente problemas y así exponer soluciones para modificar procedimientos.

Para ajustar las variaciones en la combinación de casos mixtos se recomienda presentar tasas de ISQ ajustadas al riesgo, además de las tasas brutas. ${ }^{14}$ El método de ajuste de riesgo más utilizado es el índice de riesgo NNIS, cuyo objetivo es predecir la aparición de una ISQ en un paciente determinado. ${ }^{11}$ Este índice de riesgo se ha actualizado e incluye factores específicos del procedimiento que mejoran su poder predictivo.

Algunos sistemas de vigilancia reportan índices de infección estandarizados, que es el índice entre las tasas de infección observadas y las esperadas. ${ }^{15,16}$ Una relación superior a 1.0 indica que se produjeron más ISQ de lo esperado, mientras que una relación inferior a 1.0 indica lo contrario. ${ }^{11}$ La manera más 
simple de calcular el número esperado de ISQ es multiplicando el número de operaciones en cada categoría de procedimiento por la tasa de ISQ y dividiendo por 100. Esto explica la combinación de casos y, por lo tanto, es una medida resumida ajustada al riesgo. ${ }^{11}$

\section{Factores de riesgo de la infección del sitio quirúrgico}

Las ISQ de la cirugía ortopédica son complicaciones desafortunadas, cuya morbilidad representa una carga considerable para los pacientes y el sistema de salud. En la actualidad, la tasa de infección en la reducción abierta y fijación interna de fracturas es de 1 a $3 \%$ en general: $;{ }^{17}$ mientras que las fracturas de mayor energía en regiones de riesgo, incluyendo la meseta tibial, el pilón tibial y calcáneo, pueden tener tasas de infección de hasta $50 \%$ en algunos casos. ${ }^{11,18-20}$

Aparte de las implicaciones financieras de las ISQ, la infección postoperatoria ha demostrado que afecta negativamente al limitar la función de los pacientes y la salud mental, disminuyendo su calidad de vida general y aumentando la mortalidad. ${ }^{21,22}$

La capacidad de predecir qué pacientes están en riesgo de desarrollar ISQ es de interés clínico, ya que puede ser un primer paso para prevenir la infección, tal vez evitando la cirugía en situaciones con opciones de tratamiento no quirúrgico razonables o promoviendo medidas profilácticas y alterando las estrategias de manejo. ${ }^{11,23}$

Es importante identificar factores modificables de los no modificables para las cirugías electivas con la finalidad de poder limitar la presenciad de ISQ en los pacientes que no representen un riesgo quirúrgico:

- Factores de riesgo modificables que son contraindicaciones absolutas: VIH no tratado, glucosa sérica $\geq 200 \mathrm{mg} / \mathrm{dL}$, infección articular activa (en caso de colocación de prótesis), inyecciones intraarticulares dentro de los tres meses previos, uso activo de drogas intravenosas, súper obesidad (IMC $\geq 50 \mathrm{~kg} / \mathrm{m}$ ).

- Factores de riesgo modificables que son contraindicaciones relativas: obesidad, $\mathrm{HbA1c}$ elevada, tabaquismo, pacientes con alto riesgo de caídas, cáncer no metastásico, desnutrición, hepatitis C.

- Factores de riesgo no modificables que son contraindicaciones absolutas: hipertensión arterial pulmonar.

- Factores de riesgo no modificables que son contraindicaciones relativas: sexo, edad, hemiparesia, cáncer metastásico, trastornos de coagulación de la sangre, hemofilia, enfermedad de von Willebrand, infección previa de la articulación operatoria, trasplante de hígado, trasplante de riñón, hepatitis B.

\section{CONCLUSIONES}

La vigilancia de la ISQ debe ser una parte integral de los hospitales. Se deben interpretar los datos de forma adecuada, especialmente al hacer comparaciones, debido a una probable heterogeneidad de definiciones utilizadas, métodos de vigilancia, estratificación de riesgos e informes. 


\section{Los pacientes con factores de riesgo que se puedan modificar y con cirugía} no urgente deberán recibir tratamiento para intentar corregir dichos factores, con la finalidad de disminuir la ISQ. En los casos en los que no se puedan modificar los factores de riesgo, se podrán hacer estimados para la predicción de tener una ISQ y comentarlo a los pacientes y familiares.

\section{BIBLIOGRAFÍA}

1. Defrances CJ, Lucas CA, Buie VC, Golosinskiy A. 2006 National Hospital Discharge Survey. Natl Health Stat Report. 2008; (5): 1-20.

2. Meijs AP, Ferreira JA, DE Greeff SC, Vos MC, Koek MB. Incidence of surgical site infections cannot be derived reliably from point prevalence survey data in Dutch hospitals. Epidemiol Infect. 2017; 145 (5): $970-980$.

3. Astagneau P, Rioux C, Golliot F, Brücker G, Group INS. Morbidity and mortality associated with surgical site infections: results from the 1997-1999 INCISO surveillance. J Hosp Infect. 2001; 48 (4): 267-274.

4. Freeman R. Evolution of healthcare-associated infection surveillance in England: Initiatives, implementation and opportunities for innovation. BMC Proceedings. 2011; 5 (Suppl 6): 1-1.

5. King C, Aylin P, Chukwuemeka A, Anderson J, Holmes A. Assessing data sources for sustainable and continuous surveillance: surgical site infections following coronary artery bypass grafts in England. $J$ Hosp Infect. 2013; 84 (4): 305-310.

6. Tanner J, Padley W, Kiernan M, Leaper D, Norrie P, Baggott R. A benchmark too far: findings from a national survey of surgical site infection surveillance. J Hosp Infect. 2013; 83 (2): 87-91.

7. Haley RW. The scientific basis for using surveillance and risk factor data to reduce nosocomial infection rates. J Hosp Infect. 1995; 30 Suppl: 3-14.

8. Lee JT. Contemporary wound infection surveillance issues. New Horiz. 1998; 6 (2 Suppl): S20-9.

9. Roy MC, Perl TM. Basics of surgical-site infection surveillance. Infect Control Hosp Epidemiol. 1997; 18 (9): 659-668.

10. Horan TC, Gaynes RP, Martone WJ, Jarvis WR, Emori TG. CDC definitions of nosocomial surgical site infections, 1992: a modification of CDC definitions of surgical wound infections. Infect Control Hosp Epidemiol. 1992; 13 (10): 606-608.

11. Culver DH, Horan TC, Gaynes RP, Martone WJ, Jarvis WR, Emori TG, et al. Surgical wound infection rates by wound class, operative procedure, and patient risk index. National Nosocomial Infections Surveillance System. Am J Med. 1991; 91 (3B): 152S-157S.

12. Cardo DM, Falk PS, Mayhall CG. Validation of surgical wound surveillance. Infect Control Hosp Epidemiol. 1993; 14 (4): 211-215.

13. Dicks KV, Lewis SS, Durkin MJ, Baker AW, Moehring RW, Chen LF, et al. Surveying the surveillance: surgical site infections excluded by the January 2013 updated surveillance definitions. Infect Control Hosp Epidemiol. 2014; 35 (5): 570-573.

14. O'Neill $\mathrm{E}$, Humphreys $\mathrm{H}$. Use of surveillance data for prevention of healthcare-associated infection: risk adjustment and reporting dilemmas. Curr Opin Infect Dis. 2009; 22 (4): 359-363.

15. Rioux C, Grandbastien B, Astagneau P. The standardized incidence ratio as a reliable tool for surgical site infection surveillance. Infect Control Hosp Epidemiol. 2006; 27 (8): 817-824.

16. Gaynes RP, Culver DH, Horan TC, Edwards JR, Richards C, Tolson JS. Surgical site infection (SSI) rates in the United States, 1992-1998: the National Nosocomial Infections Surveillance System basic SSI risk index. Clin Infect Dis. 2001; 33 Suppl 2: S69-77.

17. Edwards JR, Peterson KD, Mu Y, Banerjee S, Allen-Bridson K, Morrell G, et al. National Healthcare Safety Network (NHSN) report: data summary for 2006 through 2008, issued December 2009. Am J Infect Control. 2009; 37 (10): 783-805.

18. Paryavi E, Stall A, Gupta R, Scharfstein DO, Castillo RC, Zadnik M, et al. Predictive model for surgical site infection risk after surgery for high-energy lower-extremity fractures: development of the risk of infection in orthopedic trauma surgery score. J Trauma Acute Care Surg. 2013; 74 (6): 1521-1527.

19. Torbert JT, Joshi M, Moraff A, Matuszewski PE, Holmes A, Pollak AN, et al. Current bacterial speciation and antibiotic resistance in deep infections after operative fixation of fractures. J Orthop Trauma. 2015; 29 (1): 7-17.

20. Perencevich EN, Sands KE, Cosgrove SE, Guadagnoli E, Meara E, Platt R. Health and economic impact of surgical site infections diagnosed after hospital discharge. Emerg Infect Dis. 2003; 9 (2): 196-203. 
21. Whitehouse JD, Friedman ND, Kirkland KB, Richardson WJ, Sexton DJ. The impact of surgical-site infections following orthopedic surgery at a community hospital and a university hospital: adverse quality of life, excess length of stay, and extra cost. Infect Control Hosp Epidemiol. 2002; 23 (4): 183-189.

22. Partanen J, Syrjälä $H$, Vähänikkilä $H$, Jalovaara P. Impact of deep infection after hip fracture surgery on function and mortality. J Hosp Infect. 2006; 62 (1): 44-49.

23. Van Walraven C, Musselman R. The Surgical Site Infection Risk Score (SSIRS): a model to predict the risk of surgical site infections. PLoS One. 2013; 8 (6): e67167. 